Audio-Tokens: a toolbox for rating, sorting and comparing audio samples in the browser.

1 Cognitive Neuroscience Unit, Montreal Neurological Institute, McGill University,

5 Montreal, QC, H3A 2B4, Canada

${ }^{2}$ Centre for Research on Brain, Language and Music, McGill University, Montreal, QC,

7 H3G 2A8, Canada

${ }^{3}$ Ernst Strüngmann Institute for Neuroscience in Cooperation with Max Planck Society,

960528 Frankfurt am Main, Germany

*Correspondence: peter.donhauser@esi-frankfurt.de, denise.klein@mcgill.ca

Acknowledgements:

This research was undertaken thanks in part to funding from the Canada First Research

14 Excellence Fund, awarded to McGill University for the Healthy Brains for Healthy Lives initiative.

15 The work was also supported by the CRBLM which is funded by the Government of Quebec via

16 the Fonds de Recherche Nature et Technologies and Société et Culture, a Discovery grant from

17 the Natural Sciences and Engineering Research Council of Canada to DK, and a fellowship to PD

18 by the Richard \& Edith Strauss foundation. We thank the members of the Klein lab in Montreal

19 and the Poeppel lab in Frankfurt for their valuable suggestions on this project. 
Abstract

Here we describe a Javascript toolbox to perform online rating studies with auditory

22 material. The main feature of the toolbox is that audio samples are associated with visual tokens

23 on the screen that control audio playback and can be manipulated depending on the type of rating.

24 This allows the collection of single- and multi-dimensional feature ratings, as well as categorical

25 and similarity ratings. The toolbox (github.com/pwdonh/audio_tokens) can be used via a plugin

26 for the widely-used jsPsych, as well as using plain Javascript for custom applications. We expect

27 the toolbox to be useful in psychological research on speech and music perception, as well as for

28 the curation and annotation of datasets in machine learning.

29 Keywords: Online experiments, Auditory perception, Rating, Javascript

30

31 
TOOLBOX FOR RATING AUDIO SAMPLES

Audio-Tokens: a toolbox for rating, sorting and comparing audio samples in the browser.

Collecting ratings from human participants is commonplace in behavioural research at

34 multiple steps within a project. This includes validation and annotation of items to be included in

35 a corpus (Ardila et al., 2019; Belin, Fillion-Bilodeau, \& Gosselin, 2008; Paquette, Peretz, \& Belin,

36 2013) or stimulus material for neuroimaging experiments (Charest, Kievit, Schmitz, Deca, \&

37 Kriegeskorte, 2014; Gold et al., 2019); but also to test hypotheses, such as perceptual differences

38 between groups of participants (Atagi \& Bent, 2016; Jack, Garrod, Yu, Caldara, \& Schyns, 2012;

39 Kutlu, Tiv, Wulff, \& Titone, 2020) or the effect of experimental conditions and stimulus properties

40 (Holz, Larrouy-Maestri, \& Poeppel, 2021; Lavan, Scott, \& McGettigan, 2016; McDermott, Lehr,

41 \& Oxenham, 2010; Thoret, Caramiaux, Depalle, \& McAdams, 2020).

In research on speech and music, a common way to perform ratings is to first present an

43 audio stimulus and subsequently present one or multiple rating scales (Belin et al., 2008; Holz et

44 al., 2021). This sequential procedure requires the participant to hold the stimulus in working

45 memory to perform the rating. This can be demanding for the participant, especially if multiple

46 rating scales are used, or multiple audio stimuli are required to be rated at once. To alleviate this,

47 a rating interface should allow the participant to control the playback of audio stimuli. At the same

48 time, it is important that there is clear correspondence between playback controls and rating

49 controls for individual audio stimuli, such as the multiple interfaces we outline below.

50 We present multiple rating interfaces in this paper that follow these constraints. They were

51 inspired by two examples in the literature. The first one is called "Auditory free classification"

52 (Clopper, 2008), which has been applied to non-native speech perception (Atagi \& Bent, 2013,

53 2016). Participants are presented with a grid and multiple icons that control playback of audio

54 stimuli from different speakers. These icons have to be arranged in the grid, forming as many 
55 groups of speakers as the participant thinks there are. The second example is based on a study on

56 voice classification (Lavan et al., 2020) where participants were given a PowerPoint slide with

57 embedded audio samples (represented as icons). They had to sort the icons on the slides into

58 identities by clicking and dragging them. Instructions were either to sort them into two identities

59 or choose the number of identities by themselves. Both these examples show creative ways of

60 integrating audio playback controls in the rating/sorting interface. Here we expand on these ideas

61 to generate rating interfaces that span a wide range of rating types. We implemented these in a

62 toolbox called Audio-Tokens, which is easy to use and can be integrated within existing

63 experimental workflows (both online and in person studies).

64

68 playback of the audio sample by hovering over the corresponding token (Fig. 1B). Ratings are

69 performed by dragging tokens to different positions within an arena (here a rectangular box, Fig.

70 1C-D). In this paper, we introduce several rating types that are implemented in the toolbox by

71 discussing some specific examples. The aim is to encourage researchers to use the tools in their 72 own research.

\section{Continuous ratings}

One feature. This first example (Fig. 1) shows the case of rating audio samples along one specified feature. This is likely one of the most common tasks a researcher would ask from

77 participants. An example from speech research would be accent judgments. Participants are asked 
to listen to native or non-native speakers of a language and rate the strength of the foreign accent

79 they perceive; either to study the process of second language learning with a focus on the speaker

80 (Berken, Gracco, Chen, \& Klein, 2016; Flege, Munro, \& MacKay, 1995) or social factors that

81 determine what people consider 'accented' or standard variants of a language (Kutlu et al., 2020).

82 The rating is implemented by dragging tokens within the arena horizontally (left-to-right position:

83 low-to-high rating). When all tokens have been placed in the arena, participants are able to submit

84 the ratings using a button.

A common problem in many studies that use sequential presentations of stimuli for rating,

86 is that participants might slowly drift in their ratings based on the stimuli presented before (Gerratt,

87 Kreiman, Antonanzas-Barroso, \& Berke, 1993). The advantage of our approach is that it allows

88 the simultaneous rating of multiple audio stimuli. As a consequence, participants can base their

89 ratings on comparisons between the different stimuli. With the current method, a batch of speakers

90 is presented at a time, thereby the researcher can control more tightly the context of a given rating.

91 For example, the same speaker can be presented in multiple randomly selected batches of speakers

92 to average out the effects of context. In the current paper we are not trying to evaluate these effects

93 of context, but we provide the tools to enable such studies.

94 The other rating interfaces in the toolbox follow the same principle. Different rating types

95 are implemented by changing the arena layout and placement of tokens, constraining the dragging

96 operations, as well as adding lines to represent connections between different tokens. 

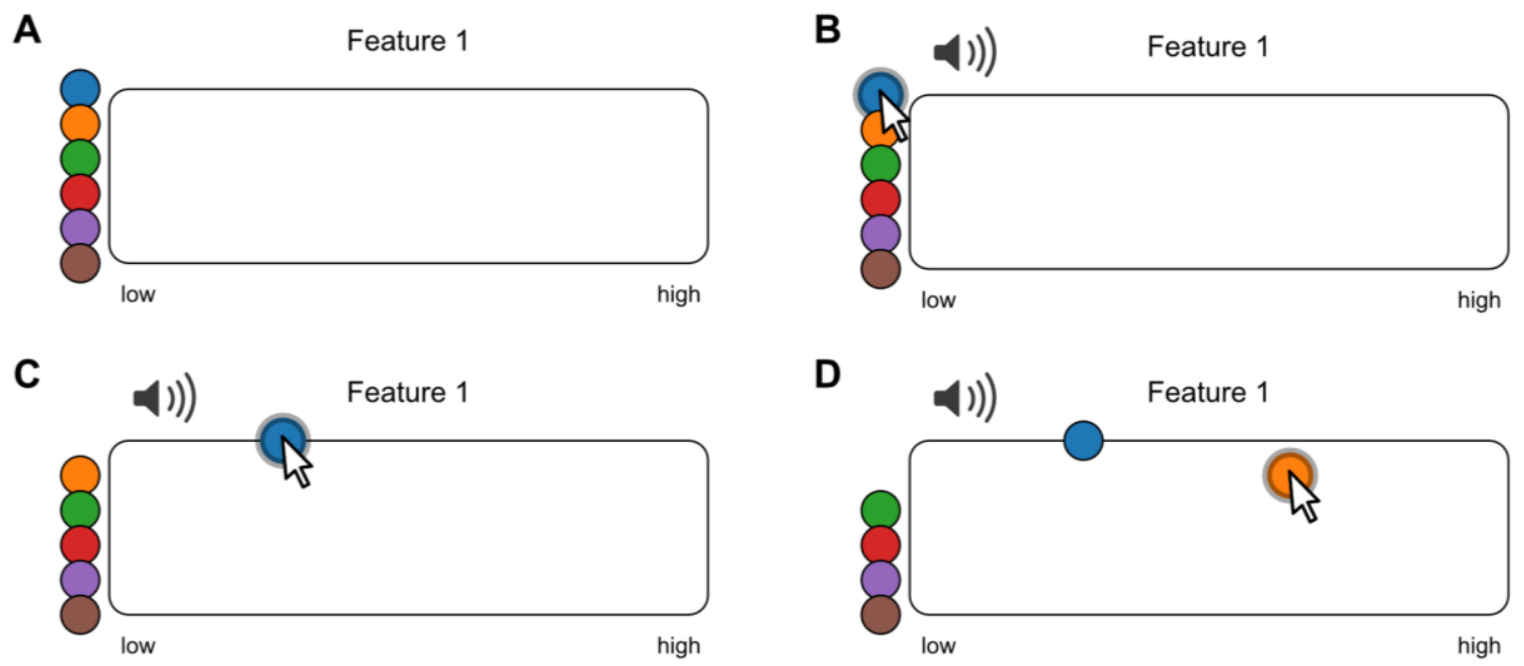

99 Figure 1: Rating audio samples along one feature. A) Multiple coloured circles (tokens) are

100 placed next to a rectangular arena. B) Each token represents an audio stimulus which is played

101 when hovering with the mouse. C-D) Tokens can be dragged horizontally to place a rating. In the

102 jsPsych plugin, use rating type: features. Use the following link for an interactive version:

103 https://pwdonh.github.io/audio_tokens/index_query.html?type=single feature

Multiple features. The next interface allows for collecting ratings along multiple feature dimensions. An example of this would be rating clinically relevant features of speech impairments, e.g. Voice quality, Articulation and Prosody (Darley, Aronson, \& Brown, 1969; Mollaei, Shiller, Baum, \& Gracco, 2016). In the example shown in Fig. 2 there are three different arenas, which

109 correspond to three feature dimensions. Tokens with the same colour represent the same audio file.

110 Just like in the previous example, participants can drag tokens horizontally to place a rating. While

111 hovering over a token, lines will appear that connect corresponding tokens (Fig. 2B). Again, in 112 order to submit the ratings, all tokens have to be dragged into the arena. 
A

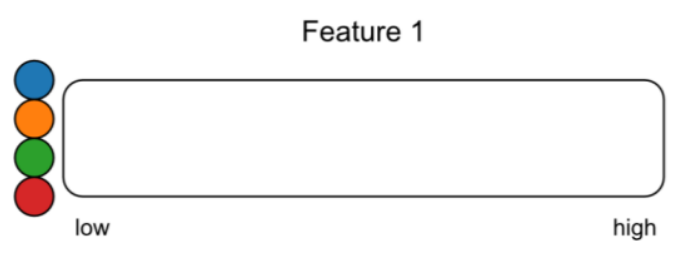

Feature 2

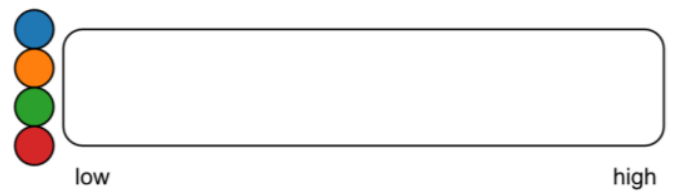

Feature 3

114

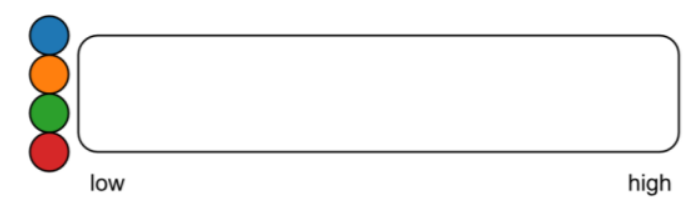

B
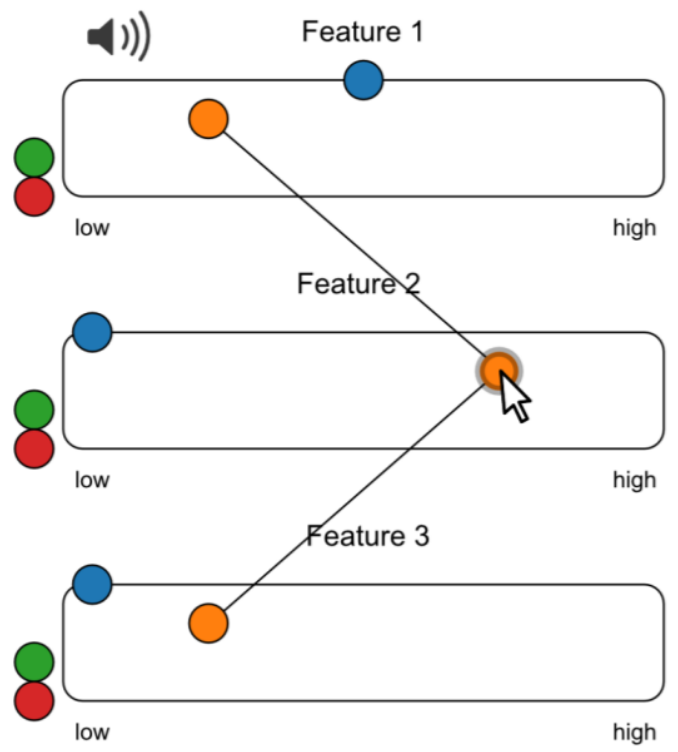

115 Figure 2: Rating audio samples along multiple features. A) Three arenas are displayed

116 corresponding to three different features to be rated. Tokens representing the same audio stimulus

117 share colour and relative position. B) Tokens can be individually dragged to place ratings for the

118 different features. Lines will appear that connect corresponding tokens. In the jsPsych plugin, use

119 rating type: features, while specifying multiple feature labels. Use the following link for an 120 interactive version:

121 https://pwdonh.github.io/audio_tokens/index_query.html?type=multiple_feature

Rating audio stimuli along multiple dimensions could be demanding for participants in a

124 sequential setup. In the interface offered here, participants can re-play stimuli multiple times, and

125 are helped by the matching colour and relative position of corresponding tokens. The connecting

126 lines are an additional visual aid: instead of the average rating they emphasise the profile across

127 the rated features, e.g. the profile of a patients' speech symptoms in the case of clinical speech

128 evaluations. 


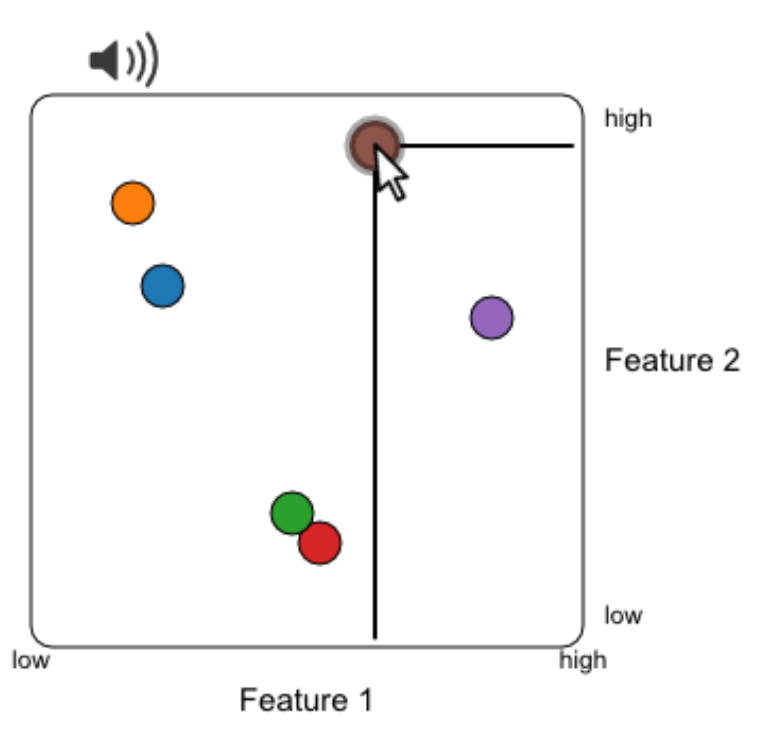

Figure 3: Rating audio samples along two features. Two dimensional feature ratings can be

131 placed in a 2D layout. In the jsPsych plugin, use rating type: features $2 \mathrm{~d}$. Use the following link

132 for an interactive version:

133 https://pwdonh.github.io/audio_tokens/index_query.html?type=features $2 \mathrm{~d}$

135 As a special case, the plugin allows ratings along two features to be performed in one arena (Fig.

136 3), by allowing both horizontal and vertical dragging operations. An example would be rating

137 emotional content of speech or music along the dimensions Valence and Arousal (Holz et al., 2021;

138 Paquette et al., 2013). Lines appear during dragging operations as a visual aid: they connect the 139 current token to the corresponding positions on the vertical and horizontal axis.

\section{Categorical ratings}

142 Fixed number of categories. Some research questions might require participants to sort 143 audio recordings into discrete categories. In one scenario the categories are specified by the 144 experimenter, for example in quality control (keep or reject) or voice identity sorting (Lavan et al., 145 2020): participants are asked to sort utterances recorded from two different speakers. In this 
TOOLBOX FOR RATING AUDIO SAMPLES interface, several tokens will appear above two arenas (here labelled as 'Category 1' and 'Category

147 2', Fig. 4A-B). Tokens can be placed in the two arenas representing the two categories. The 148 interface does not record where in the arena the tokens are dropped, because this is a discrete 149 rating. Categories can be labelled as shown below, or they can be left blank if the question is less 150 constrained ("Please sort these recordings into two piles.").
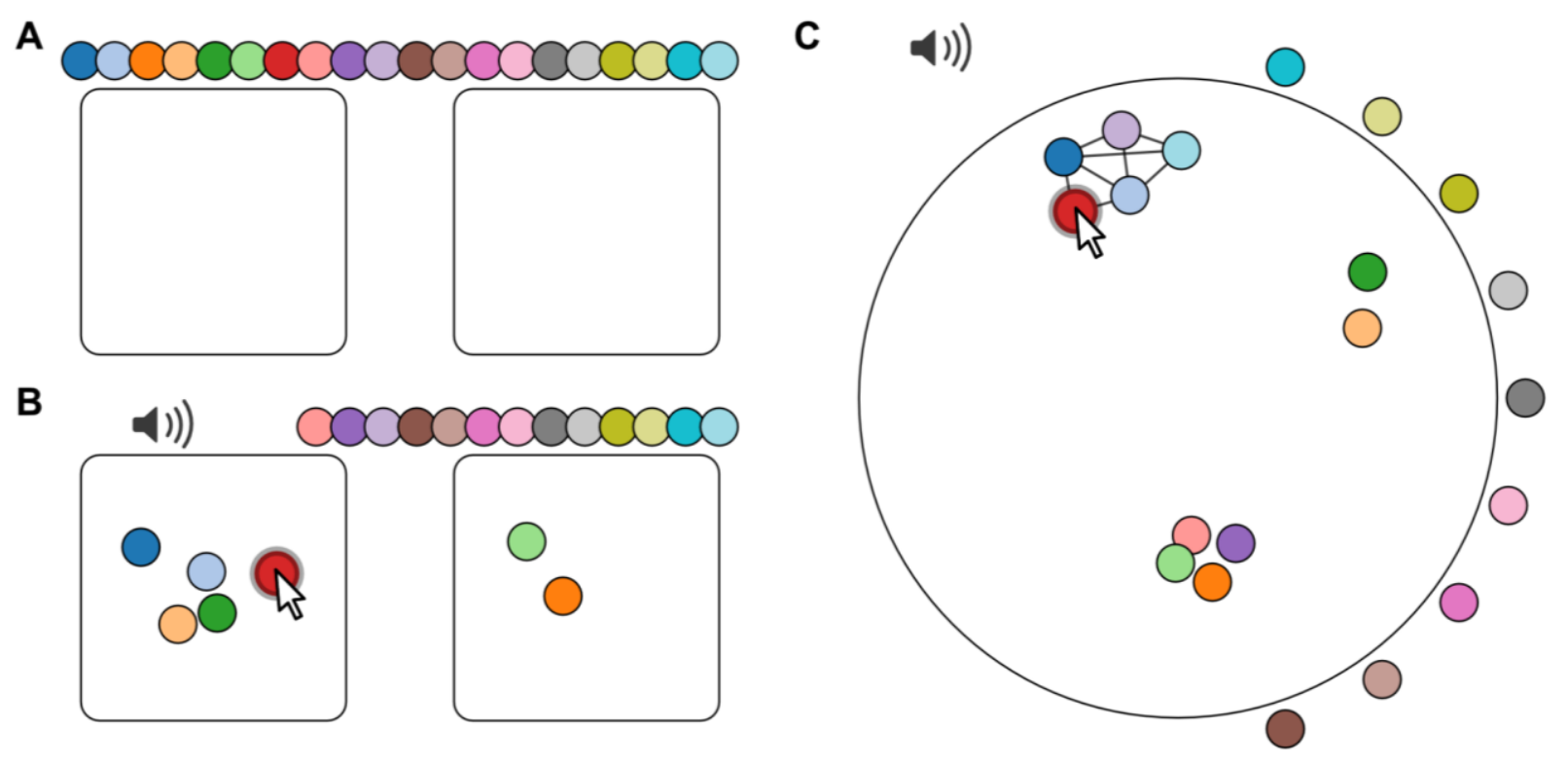

153 Figure 4: Sorting audio samples. A) Fixed number of categories: Tokens are placed on top of

154 two arenas representing two categories. B) Tokens can be sorted by dragging them into one of the 155 arenas. In the jsPsych plugin, use rating type: categories. C) Variable number of categories: 156 Tokens are placed around a circular arena and can be dragged freely inside it. Clusters are formed

157 dynamically when tokens are dragged close to each other, highlighted by connecting lines. In the 158 jsPsych plugin, use rating type: cluster. Use the following links for interactive versions:

159 https://pwdonh.github.io/audio_tokens/index_query.html?type=categories

160 https://pwdonh.github.io/audio_tokens/index_query.html?type=cluster 
TOOLBOX FOR RATING AUDIO SAMPLES

161

Variable number of categories. In another scenario, both the number of categories and

163 their labels are kept unspecified. Participants are asked to sort the recordings into as many

164 categories as deemed appropriate. For example Bent and colleagues (Bent, Atagi, Akbik, \&

165 Bonifield, 2016) asked participants to group speakers of different regional or non-native accents

166 by perceived origin.

167 In this interface (Fig. 4C), tokens are placed around a circular arena, and can be dragged

168 freely within it. Clusters are formed dynamically when tokens are placed close to each other: all

169 tokens of the current cluster are highlighted by connecting lines. Again, the interface will record

170 only the category (cluster) membership and not the exact placement of the tokens, because this is

171 a discrete rating.

172

173

\section{Similarity ratings}

174

Unconstrained similarity rating. As the most unconstrained form of rating, we provide

175 an interface for similarity ratings where participants are free to place tokens in a circular arena

176 (Fig. 5A), similar to what has been used in the visual domain (Charest et al., 2014; Kriegeskorte

$177 \&$ Mur, 2012). In contrast to the interface described above, the actual placement coordinates are

178 recorded in the results. In addition, connecting lines are drawn between the currently dragged token

179 and all others while stroke width is scaled by the distance to the other tokens: this serves as a visual

180 analogue for stimulus similarity. 


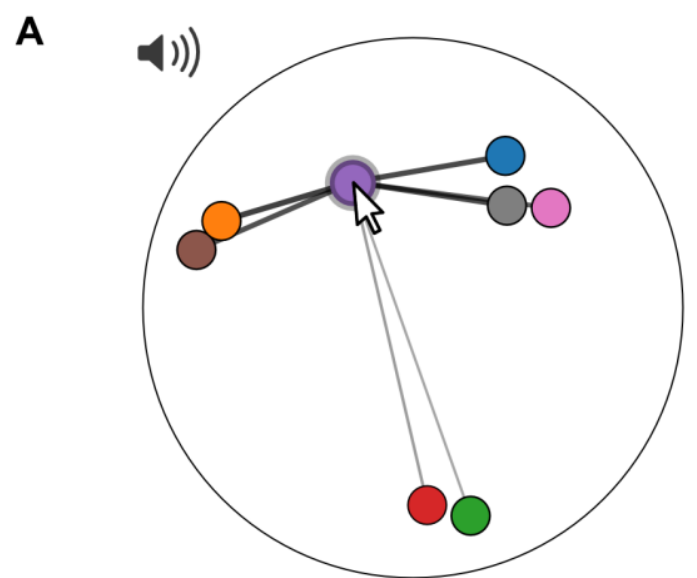

\section{B}

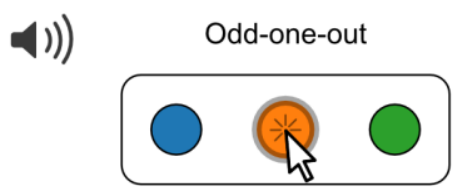

183 Figure 5: Rating the similarity of audio samples. A) Unconstrained similarity rating: The 184 interface looks similar to Fig. 4C, however the actual position of tokens is recorded rather than 185 category membership. Connecting lines emphasize the distance between tokens via the stroke width. In the jsPsych plugin, use rating type: similarity. B) Relative similarity rating: Three 187 tokens are displayed at a time. Participants are asked to select the audio stimulus that is least similar to the other two by clicking on the correspoding token. In the jsPsych plugin, use rating type: triplets. Use the following links for interactive versions:

190 https://pwdonh.github.io/audio_tokens/index_query.html?type=similarity

191 https://pwdonh.github.io/audio_tokens/index_query.html?type=triplets

194 judgements on relative similarity (Anikin, Bååth, \& Persson, 2018; Hebart, Zheng, Pereira, \& 195 Baker, 2020; Raijmakers, Jansen, \& Maas, 2004): Out of three stimuli, which one is the least 196 similar to the other two (the odd-one-out). This interface differs from the others, since there are no 
dragging operations. Instead, three tokens are shown on the screen at a given time and the odd-

198 one-out is selected by a button click. If a trial consists of only three stimuli, it ends here. If there

199 are more than three stimuli, the selected token will disappear and a new token will appear at its

200 place. The participant can make a new decision: which one is now the odd-one-out? Since the

201 decisions are made by mouse click the trial ends automatically and there is no submit button.

202 A similarity rating as in Fig. 5A can be demanding for the participant, especially as the

203 number of tokens increases. Stimuli might differ on many dimensions, but the two-dimensional

204 layout will require participants to ignore some of those dimensions. For placement of a token,

205 participants have to simultaneously consider the similarity of the current to all the other stimuli,

206 which could be highly demanding. The second task (Fig. 5B) is deliberately much simpler: the

207 participant has to compare only 3 different stimuli and make a discrete judgement. This is a less

208 expressive type of rating, but might be much easier and faster for participants to perform.

\section{Usage of the toolbox}

\section{Implementation options}

The basic tools are written in Javascript, because it is the predominant scripting language

213 for the Web. The graphical elements are drawn in the browser as svg using the data visualization

214 library $\mathrm{d} 3^{1}$. The rating tools can thus be flexibly used in any type of environment (e.g. personal or

215 institutional web page). We tested functionality for Chrome and Firefox browsers.

216 More convenient than using plain Javascript, we implemented the toolbox as a plugin

217 within the widely used jsPsych framework for running online experiments (de Leeuw, 2015). This

218 means our tools can be easily integrated in existing behavioural experiments running on jsPsych.

\footnotetext{
${ }^{1}$ d3js.org
} 
219 There are also multiple options of hosting jsPsych experiments such as Pavlovia ${ }^{2} \mathrm{Jatos}^{3}$ and

220 others; all of which are described in the official jsPsych documentation ${ }^{4}$. Although built for online

221 experiments, all experiments written in jsPsych can be performed in a laboratory setting as well,

222 using a browser on a local computer.

223

\section{Example script for jsPsych plugin}

We go through the basic steps of setting up an experiment here. The complete experiment

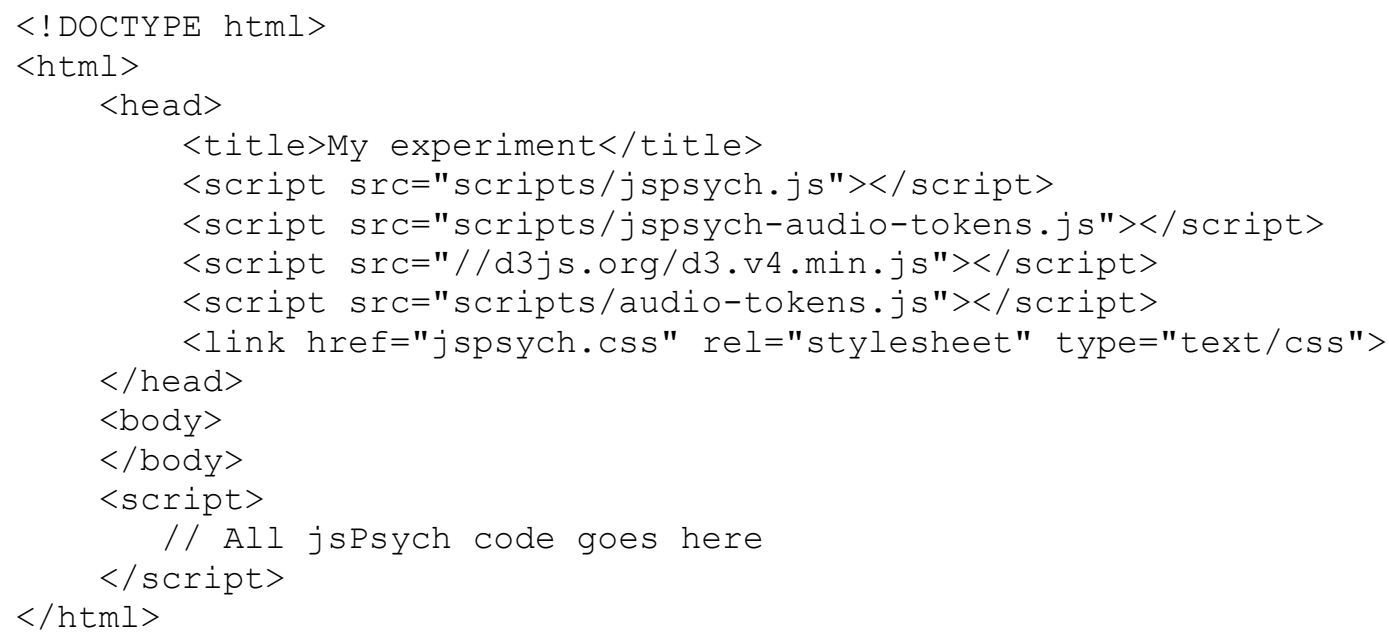

228 Here we are first importing a few libraries:

$229-\mathrm{d} 3 . \mathrm{v} 4 \cdot \mathrm{min} \cdot \mathrm{js}$ : This is the d3-library on which the current toolbox is based, it is 230 widely used for visualizing data in web environments

\footnotetext{
2 pavlovia.org

3 www.jatos.org

${ }^{4}$ jspsych.org
} 
- audio-tokens.js: This is the Javascript code for the rating tools described in this paper.

- jspsych.js: This provides all the basic functionality of jsPsych

- jspsych-audio-tokens.js: This is the plugin that allows us to include the rating tools in a jsPsych experiment

- More jsPsych plugins can be loaded here in order to build your experiment, e.g. to

There is nothing written in between the $<$ body $></$ body $>$ tags. This is normally where the content of a web page goes: here jsPsych takes care of displaying content according to the trial 240 structure of the experiment. display instructions In between the $<$ script $><$ script $>$ tags we will add all the jsPsych code:

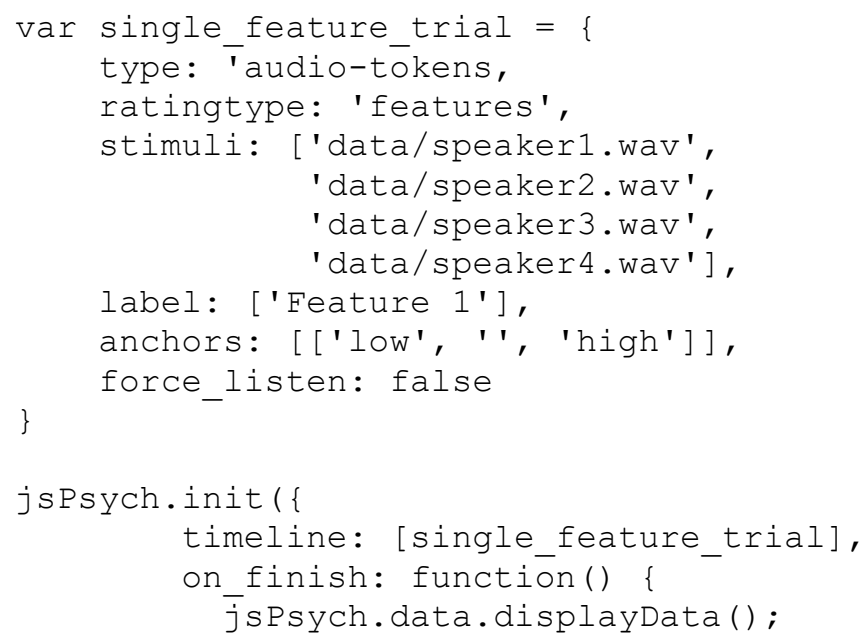

243 Here, single_feature_trial is the variable holding the parameters for a jsPsych trial:

244 - type: Here we tell jsPsych to display a trial using our plugin called 'audio-tokens' 
TOOLBOX FOR RATING AUDIO SAMPLES

- ratingtype: This tells our plugin what rating type to use among the ones described in this paper. Options are: features, features2d, categories, cluster, similarity, triplets

- stimuli: This is an array containing the file paths of the audio stimuli for this trial relative to the directory where the html file is stored.

- label: This specifies the label to be displayed for a given rating dimension (e.g. valence, arousal, accentedness). See Fig. 1, the label on top of the arena.

- anchors: This specifies the labels displayed as the endpoints of the rating dimensions (e.g. low-high, positive-neutral-negative)

- force_listen: This, if set to true, checks whether the participant has listened to the whole audio file before allowing them to submit their ratings

257 includes only one trial and the recorded data will be presented on the screen after the experiment 258 is finished. The data is by default formatted in json, and for the example trial looks like the 259 following: 


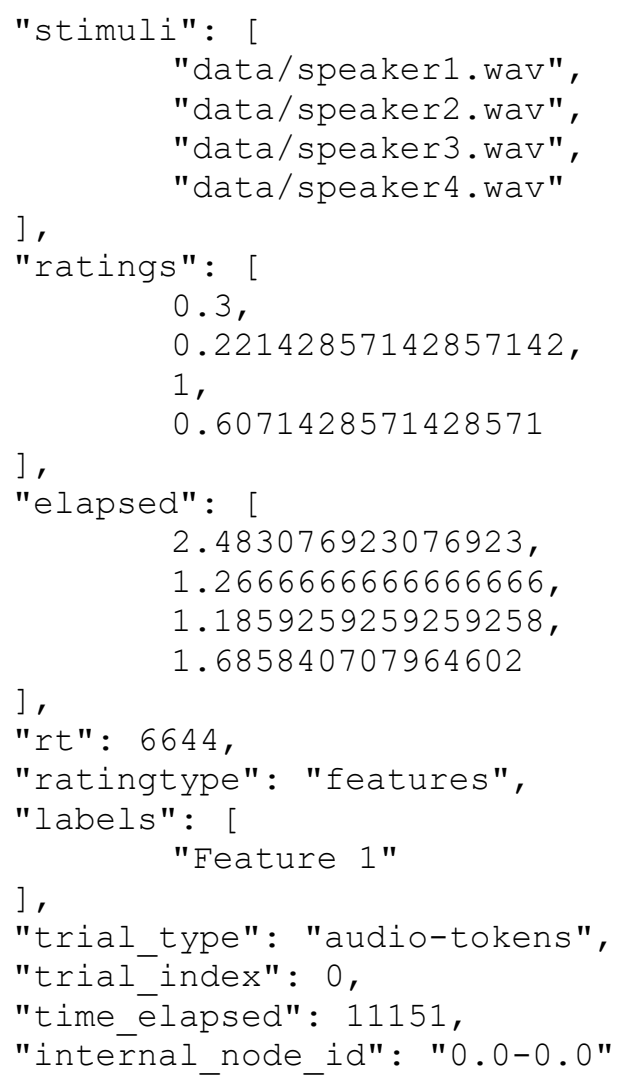

261 The following fields are specific to our plugin:

$262 \quad$ - ratings: An array containing the rating the participant gave. This differs depending 263 on the rating type. Here we get a number between 0 and 1 representing the horizontal 264 placement of each token (0: left, 1: right).

265 - elapsed: An array containing the number of times a stimulus has been played by the 266 participant. When hovering over a token, the corresponding audio stimulus is played in 267 a loop. In this example, the first stimulus was played approximately two and a half 268 times.

$269 \quad$ - rt: The time, in milliseconds, from starting the trial to submitting the ratings 
The fields stimuli, ratingtype, labels and anchors are equivalent to the

271 parameters specified in the experiment script. The remaining fields are generic jsPsych outputs.

272 Please refer to our Github repository ${ }^{5}$ for additional examples and the jsPsych

273 documentation on multiple ways to save the data. This will also depend on what solution is used

274 for hosting the experiment.

275

\section{Conclusions}

In this paper, we presented a new toolbox for collecting human ratings of audio samples

278 through the browser. We think it is unique in the range of options that are available: using a 279 consistent interface design, participants can be asked to rate audio stimuli on one or multiple

280 feature dimensions, sort audio stimuli into two or more categories, or compare sets of audio stimuli

281 using e.g. an odd-one-out procedure. As such, it adds substantial flexibility in the design of rating

282 studies, which would otherwise be constrained by the sequential nature of the task (first listen then

283 rate). In addition, it is possible to extend the toolbox to novel applications while preserving the

284 basic principle: e.g. by changing arena layout, changing token placement, setting different

285 constraints on dragging operations or allowing continuous manipulation of stimulus features

286 through dragging operations (see e.g. Harrison et al., 2020). In conclusion, we hope that

287 researchers will take advantage of the flexibility offered by the toolbox and that it can help generate

288 new experimental designs and research questions.

\footnotetext{
${ }^{5}$ github.com/pwdonh/audio_tokens
} 
TOOLBOX FOR RATING AUDIO SAMPLES

References

290

291

292

293

294

295

Anikin, A., Bååth, R., \& Persson, T. (2018). Human Non-linguistic Vocal Repertoire: Call Types and Their Meaning. Journal of Nonverbal Behavior, 42(1), 53-80.

Ardila, R., Branson, M., Davis, K., Henretty, M., Kohler, M., Meyer, J., ... Weber, G. (2019). Common Voice: A Massively-Multilingual Speech Corpus. Retrieved from http://arxiv.org/abs/1912.06670

Atagi, E., \& Bent, T. (2013). Auditory free classification of nonnative speech. Journal of Phonetics, 41(6). doi:10.1016/j.wocn.2013.09.003

Atagi, E., \& Bent, T. (2016). Auditory free classification of native and nonnative speech by nonnative listeners. Applied Psycholinguistics, 37(2), 241-263.

Belin, P., Fillion-Bilodeau, S., \& Gosselin, F. (2008). The Montreal Affective Voices: a validated set of nonverbal affect bursts for research on auditory affective processing. Behavior Research Methods, 40(2), 531-539.

Bent, T., Atagi, E., Akbik, A., \& Bonifield, E. (2016). Classification of regional dialects, international dialects, and nonnative accents. Journal of Phonetics, 58, 104-117.

Berken, J. A., Gracco, V. L., Chen, J.-K., \& Klein, D. (2016). The timing of language learning shapes brain structure associated with articulation. Brain Structure \& Function, 221(7), $3591-3600$.

Charest, I., Kievit, R. A., Schmitz, T. W., Deca, D., \& Kriegeskorte, N. (2014). Unique semantic space in the brain of each beholder predicts perceived similarity. Proceedings of the National Academy of Sciences of the United States of America, 111(40), 14565-14570.

Clopper, C. G. (2008). Auditory free classification: methods and analysis. Behavior Research Methods, 40(2), 575-581. 
TOOLBOX FOR RATING AUDIO SAMPLES

312 Darley, F. L., Aronson, A. E., \& Brown, J. R. (1969). Differential diagnostic patterns of dysarthria. Journal of Speech and Hearing Research, 12(2), 246-269.

314 de Leeuw, J. R. (2015). jsPsych: a JavaScript library for creating behavioral experiments in a Web browser. Behavior Research Methods, 47(1), 1-12.

316 Flege, J. E., Munro, M. J., \& MacKay, I. R. (1995). Factors affecting strength of perceived foreign accent in a second language. The Journal of the Acoustical Society of America, 97(5 Pt 1), $3125-3134$.

Gerratt, B. R., Kreiman, J., Antonanzas-Barroso, N., \& Berke, G. S. (1993). Comparing internal and external standards in voice quality judgments. Journal of Speech and Hearing Research, 36(1), 14-20.

Gold, B. P., Mas-Herrero, E., Zeighami, Y., Benovoy, M., Dagher, A., \& Zatorre, R. J. (2019). Musical reward prediction errors engage the nucleus accumbens and motivate learning. Proceedings of the National Academy of Sciences of the United States of America, 116(8), $3310-3315$.

Harrison, P. M. C., Marjieh, R., Adolfi, F., van Rijn, P., Anglada-Tort, M., Tchernichovski, O., ... Jacoby, N. (2020). Gibbs Sampling with People. Retrieved from http://arxiv.org/abs/2008.02595

Hebart, M. N., Zheng, C. Y., Pereira, F., \& Baker, C. I. (2020). Revealing the multidimensional mental representations of natural objects underlying human similarity judgements. Nature Human Behaviour, 4(11), 1173-1185.

332 Holz, N., Larrouy-Maestri, P., \& Poeppel, D. (2021). The paradoxical role of emotional intensity in the perception of vocal affect. Scientific Reports, 11(1), 9663. 
TOOLBOX FOR RATING AUDIO SAMPLES

Jack, R. E., Garrod, O. G. B., Yu, H., Caldara, R., \& Schyns, P. G. (2012). Facial expressions of emotion are not culturally universal. Proceedings of the National Academy of Sciences of the United States of America, 109(19), 7241-7244.

Kriegeskorte, N., \& Mur, M. (2012). Inverse MDS: Inferring Dissimilarity Structure from Multiple Item Arrangements. Frontiers in Psychology, 3, 245.

Kutlu, E., Tiv, M., Wulff, S., \& Titone, D. (2020). The Impact of Race on Speech Perception and Accentedness Judgments in Racially Diverse and Non-Diverse Groups. PsyArxiv. Retrieved from https://psyarxiv.com/hv25r/

Lavan, N., Merriman, S. E., Ladwa, P., Burston, L. F. K., Knight, S., \& McGettigan, C. (2020).

McDermott, J. H., Lehr, A. J., \& Oxenham, A. J. (2010). Individual differences reveal the basis of consonance. Current Biology: CB, 20(11), 1035-1041.

Mollaei, F., Shiller, D. M., Baum, S. R., \& Gracco, V. L. (2016). Sensorimotor control of vocal pitch and formant frequencies in Parkinson's disease. Brain Research, 1646, 269-277.

Paquette, S., Peretz, I., \& Belin, P. (2013). The "Musical Emotional Bursts": a validated set of musical affect bursts to investigate auditory affective processing. Frontiers in Psychology,

Raijmakers, M. E. J., Jansen, B. R. J., \& Maas, H. L. J. van der. (2004). Rules and development in triad classification task performance. Developmental Review: DR, 24(3), 289-321. 
357 Thoret, E., Caramiaux, B., Depalle, P., \& McAdams, S. (2020). Learning metrics on spectrotemporal modulations reveals the perception of musical instrument timbre. Nature Human Behaviour. doi:10.1038/s41562-020-00987-5 